\title{
GROUPS, GRAPHS, AND THE HANNA NEUMANN CONJECTURE
}

\author{
IGOR MINEYEV
}

AbSTRACt. Submultiplicativity, an analytic property generalizing the Strengthened Hanna Neumann Conjecture (SHNC) to complexes was proved in [2] assuming the deep-fall property. This in particular implied SHNC. The purpose of this note is to write the proof of the original SHNC and purely in terms of groups and graphs. We also give explicit examples showing that the upper bound in SHNC is sharp.

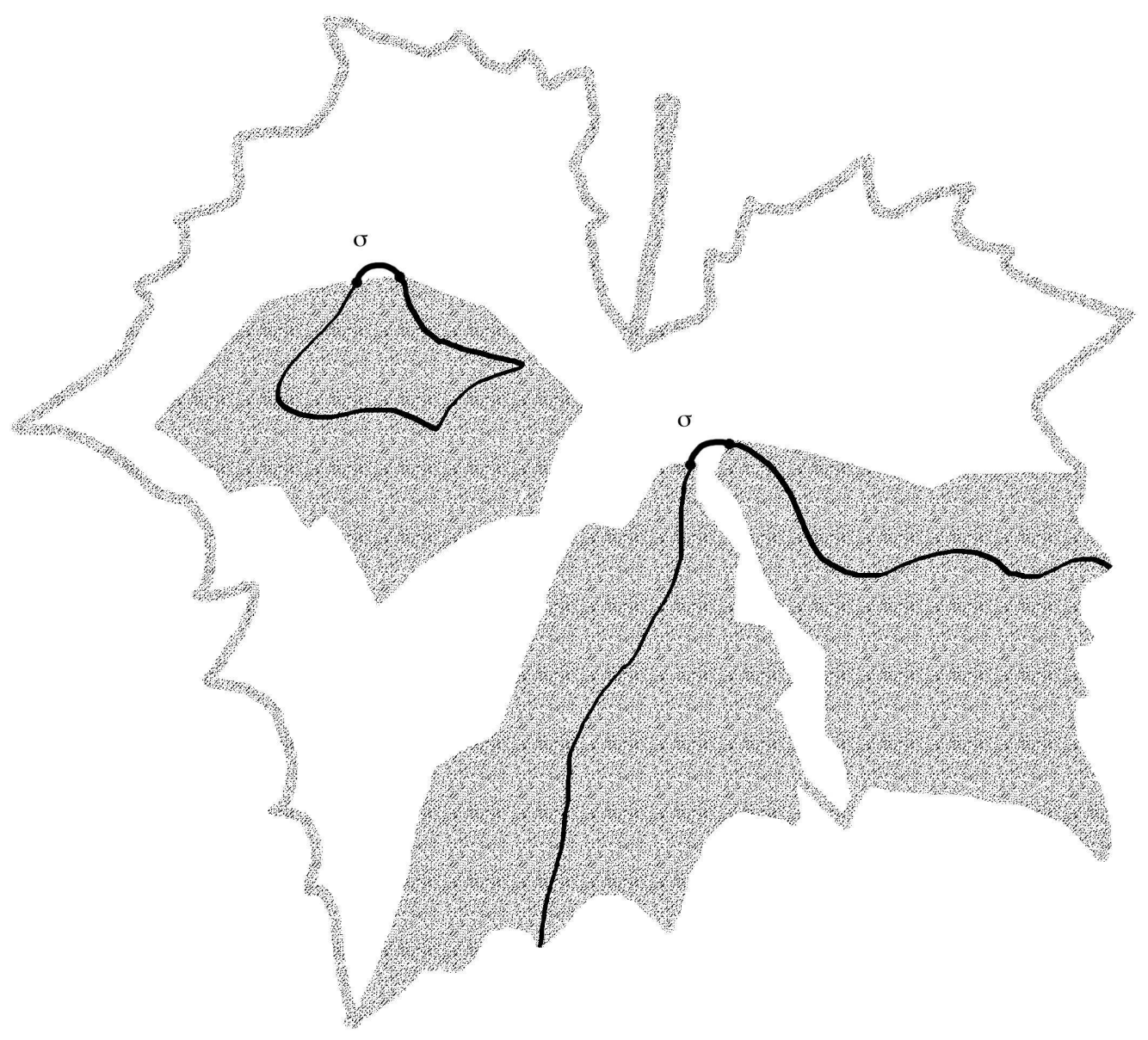

Date: May 21, 2011. Last revision December 20, 2011. 


\section{INTRODUCTION.}

The reduced rank of a free group $\Gamma$ is the number $\bar{r}(\Gamma):=\max \{0, \mathrm{rk} \Gamma-1\}$ [5, p. 162]. With this notation, the Hanna Neumann Conjecture (HNC) can be stated as follows.

Conjecture (HNC $[3,4])$. Suppose $\Gamma$ is a free group and $A$ and $B$ are its finitely generated subgroups. Then $\bar{r}(A \cap B) \leq \bar{r}(A) \cdot \bar{r}(B)$.

Walter Neumann proposed the following Strengthened Hanna Neumann Conjecture (SHNC). Let $A \backslash \Gamma / B$ be the set of all double cosets $A g B$ for $g \in \Gamma$ and $s: A \backslash \Gamma / B \rightarrow \Gamma$ be a section of the quotient map $\Gamma \rightarrow A \backslash \Gamma / B$. Denote $A^{u}:=u^{-1} A u$.

Conjecture (SHNC [5]). Suppose $\Gamma$ is a free group and $A$ and $B$ are its finitely generated subgroups. Then $\sum_{u \in s(A \backslash \Gamma / B)} \bar{r}\left(A^{u} \cap B\right) \leq \bar{r}(A) \cdot \bar{r}(B)$.

In [2], a proof of submultiplicativity for complexes was given under an additional assumption: the deep-fall property. See also references to results on SHNC there. The deep-fall property always holds for graphs, this was proved in [2] by graph-theoretic means. That in particular implied SHNC. The deep-fall property is also related to the Atiyah Conjecture.

After [2] was posted, Warren Dicks asked the author to write a purely combinatorial proof of the original SHNC. This note presents a self-contained proof of SHNC that does not use analysis; it follows the outline of [2]. We give an explicit description of leafages introduced in [1]. Leafages and left orderability are used to prove SHNC. In section 1.7 we also provide explicit examples showing that the upper bound in SHNC is sharp. Theorem 4 below and its generalization to complexes of any dimension was proved in [2, Proposition 10] by analytic means. The combinatorial proof of Theorem 4 for graphs presented below is based on the proof that Dicks subsequently sent to the author. Dicks' argument was for bi-orderable groups, the author generalized it to left-orderable groups. The author would like to thank Warren Dicks for the suggestions. A proof of SHNC has also been announced by Joel Friedman. This research is partially supported by the NSF grant DMS 07-06876. The author would also like to thank Ivan Mineyev for providing a leaf sample for the above picture.

1.1. Graphs and relative graphs. The edges in graphs will be assumed to be oriented, and all actions and graph maps will be required to preserve the orientations of edges. Let $\hat{Y}$ be any graph. Denote $V^{\hat{Y}}$ and $E^{\hat{Y}}$ the sets of vertices and edges of $\hat{Y}$, respectively. We view $\hat{Y}$ formally as the union $V^{\hat{Y}} \sqcup E^{\hat{Y}}$. For $\sigma \in E^{\hat{Y}}, \sigma^{-}$and $\sigma^{+}$denote the initial and terminal vertices of $\sigma$, respectively. $\operatorname{Comp}(Y)$ is the set of connected components of $Y$.

A path $p$ in a graph $\hat{Y}$ from a vertex $v$ to a vertex $v^{\prime}$ is a formal sequence of vertices and edges $v=v_{0}, \sigma_{1}, v_{1}, \ldots, v_{n-1}, \sigma_{n}, v_{n}=v^{\prime}$ such that $v_{i} \in V^{\hat{Y}}, \sigma_{i} \in E^{\hat{Y}}$, and $\left(\sigma_{i}^{-}=v_{i}\right.$ and $\sigma_{i}^{+}=$ $\left.v_{i-1}\right)$ or $\left(\sigma_{i}^{-}=v_{i-1}\right.$ and $\left.\sigma_{i}^{+}=v_{i}\right)$. Define infinite paths similarly. If $p$ is a path as above, the path $v^{\prime}=v_{n}, \sigma_{n}, v_{n-1}, \ldots, v_{1}, \sigma_{1}, v_{0}=v$ will be denoted $p^{-1}$.

For a subset $E \subseteq E^{\hat{Y}}$, the relative graph is $\hat{Y}(E):=V^{\hat{Y}} \sqcup E$. For a vertex $v, \hat{Y}(E, v)$ denotes the relative component, that is the connected component of $\hat{Y}(E)$ containing $v$ [2, section 6.2]. 
$\Gamma$ will be any group; we will not require $\Gamma$ to be finitely generated or ordered. A $\Gamma$-graph is a graph with a left $\Gamma$-action. A $\Gamma$-graph will be called free-edge if the induced $\Gamma$-action on $E^{\hat{Y}}$ is free. A $\Gamma$-graph is free if the $\Gamma$-action on $\hat{Y}$ is free.

1.2. Gardens and essential sets. The reduced rank of a finite graph $Y$ is the number $\bar{r}(Y):=\sum_{K \in \operatorname{Comp}(Y)} \max \{0,-\chi(K)\}$, where $\chi$ is the Euler characteristic. In particular, $\bar{r}(\emptyset)=0$. This is consistent with the definition for free groups: if $Y$ is nonempty, then $\bar{r}(Y)=\sum_{K \in \operatorname{Comp}(Y)} \bar{r}\left(\pi_{1}(K)\right)$. With a bit of work this notion can be generalized to infinite graphs, if needed.

A tree is a contractible graph. A forest is a disjoint union of trees. A flower is a graph homotopy equivalent to a circle. A garden is a disjoint union of trees and flowers [1, section 5.1].


Essential sets of edges were defined in [1, subsection 5.3]. An edge $\sigma$ in a finite graph $Y$ is called essential in $Y$ if $\bar{r}(Y \backslash\{\sigma\})=\bar{r}(Y)-1$. More generally, a set of edges $E \subseteq E^{Y}$ is called essential in $Y$ if $\bar{r}(Y \backslash E)=\bar{r}(Y)-\# E$. A maximal essential set is just what it says.

There are several characterizations of essential sets and maximal essential sets (see [1, Lemma 12]). In particular, a set $E \subseteq E^{Y}$ is maximal essential if and only if $\bar{r}(Y \backslash E)=$ $\bar{r}(Y)-\# E=0$; so for any maximal essential set $E$ we have $\bar{r}(Y)=\# E$. Also, $E \subseteq E^{Y}$ is essential in $Y$ if for each $\sigma \in E$, the component of $Y \backslash E$ containing $\sigma^{-}$is not a tree and the component of $Y \backslash E$ containing $\sigma^{+}$is not a tree. This is shown by removing edges in $E$ one by one and checking that the reduced rank of the finite graph decreases exactly by 1 at each step.

1.3. Leafages and systems. A leafage is a map between complexes whose restriction to each component in its domain is injective [2, section 4]. (The components of the domain are viewed as "leaves" as on the picture below.)

Let $\Gamma, A, B$ be as in the statement of SHNC. Let $\hat{X}$ be the Cayley graph of $\Gamma$ with respect to some basis. If $A$ is trivial, let $\mathcal{A}$ be the empty set. If $A$ is nontrivial, let $\mathcal{A}$ be any nonempty $A$-invariant subtree of $\Gamma$ such that the $A$-action on $\mathcal{A}$ is cocompact. For example, we can make some choice of a basepoint $y_{0}$ in $V^{\hat{X}}$, pick a path from $y_{0}$ to $a y_{0}$ for each $a$ in a finite generating set of $A$, and let $\mathcal{A}$ be the onion of all the $A$-translates of these paths. Since $\mathcal{A}$ is simply connected, $A$ is the fundamental group of the finite quotient $A \backslash \mathcal{A}$.

We make translates of $\mathcal{A}$ in $\hat{X}$ formally disjoint as follows. For $y \in \Gamma / A, y \mathcal{A}$ will mean the subgraph $\bar{y} \mathcal{A} \subseteq \hat{X}$, where $\bar{y}$ is any representative of the left coset $y$. The induced graph is

$$
\hat{Y}:=\{(y, \sigma) \in(\Gamma / A) \times \hat{X} \mid \sigma \in y \mathcal{A}\} .
$$


A leaf in $\hat{Y}$ for $y \in \Gamma / A$ is the graph $\hat{Y}_{y}:=\{(y, \sigma) \mid \sigma \in y \mathcal{A}\} \subseteq \hat{Y}$. $\hat{Y}$ is the disjoint union of its leaves, $\hat{Y}=\bigsqcup_{y \in \Gamma / A} \hat{Y}_{y}$. The map

$$
\hat{\alpha}: \hat{Y} \rightarrow \hat{X}, \quad \hat{\alpha}(y, \sigma):=\sigma,
$$

is a leafage. We put the diagonal $\Gamma$-action on $\hat{Y}: g(y, \sigma):=(g y, g \sigma)$ for $g \in \Gamma,(y, \sigma) \in \hat{Y}$. This turns $\hat{\alpha}$ into a $\Gamma$-leafage, i.e. a leafage commuting with the $\Gamma$-actions.

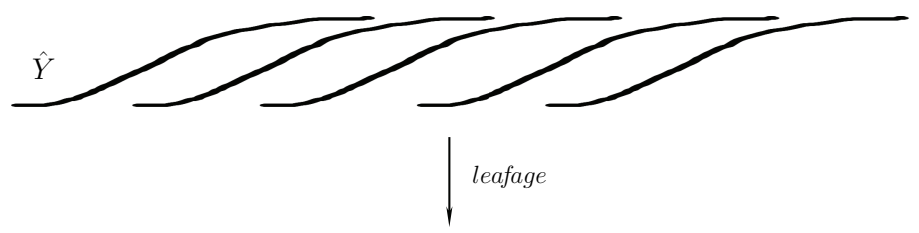

$\hat{X}$

The $\Gamma$-action on $\hat{Y}$ is free and it permutes the leaves of $\hat{Y}$, i.e. it induces a $\Gamma$-action on Leaves $(\hat{Y})$, the set of leaves of $\hat{Y}$, by the formula $g \hat{Y}_{y}=\hat{Y}_{g y}$. The stabilizers of leaves are conjugates of $A: \operatorname{Stab}_{\Gamma}\left(\hat{Y}_{y}\right)=A^{y^{-1}}$, where $A^{y^{-1}}$ is the notation for $A^{\bar{y}^{-1}}=\bar{y} A \bar{y}^{-1}$ for any choice of $\bar{y} \in y$. $\mathcal{A}$ can be identified with the leaf $\hat{Y}_{1 A}$ of $\hat{Y}$ via the inclusion $\mathcal{A} \hookrightarrow \hat{Y}, \sigma \mapsto(1 A, \sigma)$. Hence the quotient $Y:=\Gamma \backslash \hat{Y}$ is isomorphic to $A \backslash \hat{Y}_{1 A}=A \backslash \mathcal{A}$.

Similarly, for the subgroup $B \leq \Gamma$ define a $B$-invariant subtree $\mathcal{B} \subseteq \hat{X}$, the induced graph $\hat{Z}$, and the $\Gamma$-leafage $\hat{\beta}: \hat{Z} \rightarrow \hat{X}$. Let $\hat{S}$ be the fiber product

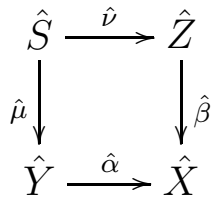

(cf. [2, section 2.2]). Explicitly, $\hat{S}=\{(y, z, \sigma) \mid(y, \sigma) \in \hat{Y},(z, \sigma) \in \hat{Z}\}, \hat{\mu}(y, z, \sigma):=(y, \sigma)$, $\hat{\nu}(y, z, \sigma):=(z, \sigma)$. For $y \in \Gamma / A$ and $z \in \Gamma / B$, let $\hat{S}_{y, z}$ be a copy of the intersection $\hat{\alpha}\left(\hat{Y}_{y}\right) \cap$ $\hat{\beta}\left(\hat{Z}_{z}\right)=y \mathcal{A} \cap z \mathcal{B} . \quad \hat{S}$ is the disjoint union of such: $\hat{S}=\bigsqcup_{y, z} \hat{S}_{y, z}$. All the arrows in the above diagram are $\Gamma$-leafages. The free $\Gamma$-actions on $\hat{X}, \hat{Y}$, and $\hat{Z}$ induce a free $\Gamma$-action on $\hat{S}$. Explicitly, $g(y, z, \sigma):=(g y, g z, g \sigma)$ for $(y, z, \sigma) \in \hat{S}, g \in \Gamma$. The $\Gamma$-action on $\hat{S}$ is free and it induces a $\Gamma$-action on Leaves $(\hat{S})$, the set of leaves of $\hat{S}$, by the formula $g \hat{S}_{y, z}=\hat{S}_{g y, g z}$. The stabilizers of leaves in $\hat{S}$ are intersections of stabilizers: $\operatorname{Stab}_{\Gamma}\left(\hat{S}_{y, z}\right)=A^{y^{-1}} \cap B^{z^{-1}}$.

Lemma 1. $\bar{r}(A \backslash \mathcal{A})=\bar{r}(A), \quad \bar{r}(B \backslash \mathcal{B})=\bar{r}(B), \quad \bar{r}\left(\left(A^{y^{-1}} \cap B^{z^{-1}}\right) \backslash(y \mathcal{A} \cap z \mathcal{B})\right)=\bar{r}\left(A^{y^{-1}} \cap B^{z^{-1}}\right)$.

Proof. If the tree $\mathcal{A}$ is nonempty, then $A$ is the fundamental group of $A \backslash \mathcal{A}$, so by the definition of reduced rank, $\bar{r}(A \backslash \mathcal{A})=\bar{r}(A)$. If $\mathcal{A}$ is empty, then $A$ is trivial by the definition of $\mathcal{A}$, so the same equality holds: $\bar{r}(A \backslash \mathcal{A})=\bar{r}(\emptyset)=0=\bar{r}(1)=\bar{r}(A)$. The equality for $B$ is similar. If $y \mathcal{A} \cap z \mathcal{B} \neq \emptyset$, the third equality holds by the same argument. If $y \mathcal{A} \cap z \mathcal{B}=\emptyset$, then $A^{y^{-1}} \cap B^{z^{-1}}$ is trivial; otherwise there would be a nontrivial element $g \in A^{y^{-1}} \cap B^{z^{-1}}$, and therefore the axis 
of $g$ in $\hat{X}$ would be contained in $y \mathcal{A} \cap z \mathcal{B}$. Hence the equality holds in this empty case as well: $\bar{r}\left(\left(A^{y^{-1}} \cap B^{z^{-1}}\right) \backslash(y \mathcal{A} \cap z \mathcal{B})\right)=\bar{r}(\emptyset)=0=\bar{r}(1)=\bar{r}\left(A^{y^{-1}} \cap B^{z^{-1}}\right)$.

Denote $S:=\Gamma \backslash \hat{S}$. This gives the system of graphs



as in [1, sections 3.1 and 3.6]. The diagonal arrows are $\Gamma$-quotient maps. $S$ is the fiber product of $Y$ and $Z$ over $X$; such fiber products of finite graphs were considered by Stallings [7].

To prove SHNC, we will make a consistent choice of maximal essential sets in $Y, Z$, and $S$ using leafages and left-invariant orders.

1.4. The fall. Let $\hat{Y}$ be a locally finite graph. Given an edge $\sigma \in E^{\hat{Y}}$ and a set of edges $E \subseteq E^{\hat{Y}} \backslash\{\sigma\}$, we say that $\sigma$ falls into $E$ if the components of the relative graph $\hat{Y}(E)$ containing $\sigma^{-}$and $\sigma^{+}$are infinite or coincide (cf. [2, Definition 2(a) and Lemma 21]). A vein at $\sigma$ in $E$ is either

(a) a path in $\hat{Y}(E)$ without self-intersections from $\sigma^{-}$to $\sigma^{+}$, called a finite vein, or

(b) a union of two disjoint infinite rays in $\hat{Y}(E)$ without self-intersections starting at $\sigma^{-}$ and $\sigma^{+}$, respectively, called an infinite vein.

It is easily checked that $\sigma$ falls into $E$ if and only if there exists a vein at $\sigma$ in $E$. If $\hat{Y}$ is a forest, all veins are infinite.

1.5. Ordered graphs. A graph $\hat{Y}$ will be called ordered if $E^{\hat{Y}}$ is given a partial order $\leq$ whose restriction to $E^{K}$ is a total order for each component $K$ of $\hat{Y}$.

An ordered $\Gamma$-graph is a $\Gamma$-graph that is ordered and such that the partial order on its edge set is preserved by the $\Gamma$-action. For example, if $\Gamma$ is a left-ordered group and $\hat{Y}$ is a free $\Gamma$-graph, we can turn $\hat{Y}$ into an ordered $\Gamma$-graph by picking any $\Gamma$-transversal subset $\bar{E}^{\hat{Y}}$ in $E^{\hat{Y}}$, putting any total order on it, and taking either lexicographic order on $E^{\hat{Y}}=\Gamma \bar{E}^{\hat{Y}} \cong \Gamma \times \bar{E}^{\hat{Y}}$. Free groups are left-orderable, and even two-sided orderable ([6, p.155-157], [8, p.165]), so the Cayley graph of a free group with respect to any basis can be viewed as a free ordered $\Gamma$-tree.

For a subset $E \subseteq E^{\hat{Y}}$ and an edge $\sigma \in E^{\hat{Y}} \backslash E$, denote $[E<\sigma]:=\{\tau \in E \mid \tau<\sigma\}$. We say that an edge $\sigma$ in $\hat{Y}$ is order-essential in $\hat{Y}$ if it falls into $\left[E^{\hat{Y}}<\sigma\right]$, and orderinessential otherwise. Note that this property is not affected if $\hat{Y}$ is replaced with its component 
containing $\sigma$. Denote by $\mathbb{E}^{\hat{Y}}$ and $\mathbb{I}^{\hat{Y}}$ the sets of order-essential and order-inessential edges in $\hat{Y}$, respectively. The following lemma is immediate.

Lemma 2. Let $\Gamma$ be any group, $\hat{Y}$ be an ordered $\Gamma$-graph, $A$ be a subgroup of $\Gamma$, and $\mathcal{A}$ be an A-invariant subgraph of $\hat{Y}$. Then

(a) $\mathcal{A}$ is an ordered $A$-graph with respect to the partial order on $E^{\mathcal{A}}$ restricted from $E^{\hat{Y}}$,

(b) $\mathbb{E}^{\mathcal{A}}$ and $\mathbb{I}^{\mathcal{A}}$ are $A$-invariant subsets of $\hat{Y}$, and

(c) $\mathbb{E}^{\mathcal{A}} \subseteq \mathbb{E}^{\hat{Y}}$.

Proposition 3 (The deep-fall property for graphs). Suppose $\hat{Y}$ is a locally-finite ordered graph. If $\sigma \in \mathbb{E}^{\hat{Y}}$, then $\sigma$ falls into $\left[\mathbb{I}^{\hat{Y}}<\sigma\right]$.

Proof. (cf. [2, lemmas 16 and 23, Theorem 24]) Since $\hat{Y}$ is locally finite, replacing $\hat{Y}$ with its component containing $\sigma$ we can assume that $\hat{Y}$ is countable, and that the order on $E^{\hat{Y}}$ is total. Since $\sigma \in \mathbb{E}^{\hat{Y}}, \sigma$ falls into $\left[E^{\hat{Y}}<\sigma\right]$. Enumerate the set $\left[\mathbb{E}^{\hat{Y}}<\sigma\right]$ as $\left\{\sigma_{1}, \sigma_{2}, \ldots\right\}$. For each $n \geq 1$, do the following. Since the order on $E^{\hat{Y}}$ is total, we can relabel the set $\left\{\sigma_{1}, \ldots, \sigma_{n}\right\}$ as $\left\{\tau_{1}, \ldots, \tau_{n}\right\}$ so that $\tau_{1}>\ldots>\tau_{n}$. Since $\tau_{1} \in \mathbb{E}^{\hat{Y}}$, then there is a vein at $\tau_{1}$ in $\left[E^{\hat{Y}}<\tau_{1}\right]$, hence in $\left[E^{\hat{Y}}<\sigma\right] \backslash\left\{\tau_{1}\right\}$. Take any vein at $\sigma$ in $\left[E^{\hat{Y}}<\sigma\right]$. If this vein contains $\tau_{1}$, replace $\tau_{1}$ with the vein at $\tau_{1}$ in $\left[E^{\hat{Y}}<\tau_{1}\right]$, then remove self-intersections if needed. The result is a subgraph of $\hat{Y}$. The components of this subgraph containing $\sigma^{-}$and $\sigma^{+}$form a vein at $\sigma$ in $\left[E^{\hat{Y}}<\sigma\right] \backslash\left\{\tau_{1}\right\}$. Repeat this for $\tau_{2}, \tau_{3}$ etc, inductively to obtain a vein at $\sigma$ in

$$
\left[E^{\hat{Y}}<\sigma\right] \backslash\left\{\tau_{1}, \ldots, \tau_{n}\right\}=\left[E^{\hat{Y}}<\sigma\right] \backslash\left\{\sigma_{1}, \ldots, \sigma_{n}\right\} .
$$

This gives a vein at $\sigma$ in $\left[E^{\hat{Y}}<\sigma\right] \backslash\left\{\sigma_{1}, \ldots, \sigma_{n}\right\}$ for each $n \geq 1$. Either this sequence of veins consists of finite veins of uniformly bounded lengths or there is a subsequence of (finite or infinite) veins at $\sigma$ whose lengths converge to $\infty$. Since $\hat{Y}$ is locally finite, all of the orderessential edges in an arbitrarily large ball around $\sigma$ will be removed at some finite stage. By taking a subsequence we can assume that either the sequence of veins stabilizes at a finite vein at $\sigma$ in $\left[E^{\hat{Y}}<\sigma\right]$ or the initial segments of those veins in $\left[E^{\hat{Y}}<\sigma\right]$ of any fixed length eventually stabilize in the sequence. In the former case there is a limiting finite vein, and in the latter case there is a limiting infinite vein. In either case, the limiting vein will be in $\left[E^{\hat{Y}}<\sigma\right] \backslash\left\{\sigma_{1}, \ldots, \sigma_{n}\right\}$ for each $n$, hence in $\left[\mathbb{I}^{\hat{Y}}<\sigma\right]$. Thus $\sigma$ falls into $\left[\mathbb{I}^{\hat{Y}}<\sigma\right]$.

Theorem 4. Let $\Gamma$ be any group and $\hat{Y}$ be a free cocompact ordered $\Gamma$-graph all of whose edges are order-inessential. Then $\hat{Y}$ is a forest and $\bar{r}(\Gamma \backslash \hat{Y})=0$.

Proof. (cf. [2, Proposition 10]) If $\hat{Y}$ is not a forest, it has a simple non-trivial loop. This loop lies in a component of $\hat{Y}$, and the order on the edges of the component is total, so there is the maximal edge $\tau$ in the loop. The rest of the loop forms a finite vein at $\tau$ in $\left[E^{\hat{Y}}<\tau\right]$, hence $\tau$ is order-essential in $\hat{Y}$, which contradicts the assumptions.

Let $\hat{Y}^{\prime}$ be a component of $\hat{Y}$ and denote $\Gamma^{\prime}:=\operatorname{Stab}\left(\hat{Y}^{\prime}\right), Y^{\prime}:=\Gamma^{\prime} \backslash \hat{Y}^{\prime}$. Then $Y^{\prime}$ is a component of $\Gamma \backslash \hat{Y}$, so it suffices to show that $\bar{r}\left(Y^{\prime}\right)=0$ (i.e. $Y^{\prime}$ is a tree or a flower). Since $\hat{Y}^{\prime}$ 
is simply connected and $\Gamma^{\prime}$ acts freely on it, then $\Gamma^{\prime}$ is the fundamental group of $Y^{\prime}$. Suppose that $\bar{r}\left(Y^{\prime}\right) \neq 0$, then $\Gamma^{\prime}$ has rank at least two. Take any two elements $g$ and $h$ of some basis in $\Gamma^{\prime}$. Let $l_{g}$ and $l_{h}$ be their axes in $\hat{Y}^{\prime}$, i.e. biinfinite periodic concatenations

$$
\ldots \cdot g^{-2} p \cdot g^{-1} p \cdot p \cdot g p \cdot g^{2} p \cdot \ldots \quad \text { and } \ldots \cdot h^{-2} q \cdot h^{-1} q \cdot q \cdot h q \cdot h^{2} q \cdot \ldots
$$

without cancellations, where $p$ and $q$ are some finite paths in $\hat{Y}^{\prime}$.

Let $E^{p}$ be the set of edges in $p$. Since $p$ lies in a component of $\hat{Y}$, the restriction of the order on $E^{\hat{Y}}$ to $E^{p}$ is total, so there is a maximal edge $\xi$ in $E^{p}$. We have $g \xi \neq \xi$ since $g$ is a basis element and the action is free. Assume that $\xi>g \xi$, then by the left-invariance of the order, ...> $>g^{-2} \xi>g^{-1} \xi>\xi>g \xi>g^{2} \xi>\ldots$. For each $\tau \in E^{p} \backslash\{\xi\}$ we have $\xi>\tau$, hence $g^{n} \xi>g^{n} \tau$ for all $n \in \mathbb{Z}$. This shows that for each $n \in \mathbb{Z}, g^{n} \xi$ is the largest edge in the ray $g^{n} p \cdot g^{n+1} p \cdot g^{n+2} p \cdot \ldots$. By removing some initial edges and cyclically relabeling the path $p$ we can assume that $g^{n} \xi$ is the initial edge in this ray. Now assume that $\xi<g \xi$, then $\cdots<g^{-2} \xi<g^{-1} \xi<\xi<g \xi<g^{2} \xi<\ldots$ and $g^{n} \xi>g^{n} \tau$ for $\tau \in E^{p} \backslash\{\xi\}$ as before. This shows that $g^{n} \xi$ is the largest edge in the ray $\ldots \cdot g^{n-2} p \cdot g^{n-1} p \cdot g^{n} p$, and we can assume that $g^{n} \xi$ is the terminal edge in it.

The same can be done for $h$ and $l_{h}$ : let $\eta$ be the maximal edge in $q$, and write similar inequalities. We assume that $\xi>g \xi$ and $\eta>h \eta$; the other three cases are similar. We have two rays

$$
g^{n} p \cdot g^{n+1} p \cdot g^{n+2} p \cdot \ldots \quad \text { and } \quad h^{k} q \cdot h^{k+1} q \cdot h^{k+2} q \cdot \ldots
$$

whose initial edges $g^{n} \xi$ and $h^{k} \eta$ are the largest edges of the two rays, respectively. Since $g$ and $h$ are different basis elements, these two rays eventually become disjoint as $n$ and $k$ increase. Connect the initial vertices of these two rays by a path, remove self-intersections and take $k$ and $n$ sufficiently large to obtain a biinfinite path $\ldots \cdot g^{n+2} p^{-1} \cdot g^{n+1} p^{-1} \cdot g^{n} p^{-1} \cdot r \cdot h^{k} q \cdot h^{k+1} q \cdot h^{k+2} q \cdot \ldots$ without cancellations, where $r$ is some finite path. Let $\omega$ be the maximal edge among the edges in $r$ and the edges $g^{n} \xi$ and $h^{k} \eta$, then every edge in the above biinfinite path other than $\omega$ is strictly smaller than $\omega$, hence $\omega$ is order-essential, which contradicts the assumptions.

Remark. Under the assumptions of Theorem 4 we can say more. The condition $\bar{r}(Y)=0$ implies that $Y$ is a finite garden. Each component $K$ of $\hat{Y}$ is the universal cover of a component of $Y$, hence $K$ must be either a finite tree with trivial stabilizer or the universal cover of a finite flower, i.e. a quasiline, with stabilizer $\mathbb{Z}$. In short, " $\hat{Y}$ universally covers a garden."

Theorem 5. Let $\Gamma$ be any group, $\hat{Y}$ be a free cocompact ordered $\Gamma$-forest, $Y:=\Gamma \backslash \hat{Y}, \mathbb{E}^{Y}:=$ $\Gamma \backslash \mathbb{E}^{\hat{Y}}$. Then $\mathbb{E}^{Y}$ is a maximal essential set in $Y$. In particular, $\bar{r}(Y)=\# \mathbb{E}^{Y}$.

Proof. (cf. [2, Lemma 28]) Denote $\mathbb{I}^{Y}:=\Gamma \backslash \mathbb{I}^{\hat{Y}}$. Take any $\sigma \in \mathbb{E}^{\hat{Y}}$. By the deep-fall property, $\sigma$ has a vein in $\left[\mathbb{I}^{\hat{Y}}<\sigma\right]$. Since $\hat{Y}$ is a forest, this vein must be infinite, hence each ray of this vein maps non-injectively into the finite quotient $Y\left(\mathbb{I}^{Y}\right)=Y \backslash \mathbb{E}^{Y}$. Since the $\Gamma$-action on $\hat{Y}$ is free, the quotient map $\hat{Y} \rightarrow Y$ is a covering, then the image of each ray in the vein via the quotient map will have at least one loop in $Y\left(\mathbb{I}^{Y}\right)$, so the component of $Y\left(\mathbb{I}^{Y}\right)$ containing $q\left(\sigma^{-}\right)$is not a tree, and similarly for $\sigma^{+}$. By the observation at the end of $1.2, \mathbb{E}^{Y}$ is essential in $Y$. 
The edges in $\mathbb{I}^{\hat{Y}}$ are order-inessential in $\hat{Y}$, hence they remain order-inessential in the smaller graph $\hat{Y}\left(\mathbb{I}^{\hat{Y}}\right)$. Theorem 4 says that $\bar{r}\left(Y\left(\mathbb{I}^{Y}\right)\right)=0$, hence $\mathbb{E}^{Y}$ is maximal in $Y$.

Remark. If $\hat{Y}$ be a free cocompact ordered $\Gamma$-graph (not necessarily a forest), then $\mathbb{E}^{Y}$ contains a maximal essential set of $Y$, so $\bar{r}(Y) \leq \# \mathbb{E}^{Y}$. This can be deduced by a similar argument.

1.6. The proof of SHNC. Consider a system as in (1). Put a $\Gamma$-invariant order on $E^{\hat{X}}$ as in 1.5. Pull back this order from $\hat{X}$ to each leaf of $\hat{Y}, \hat{Z}, \hat{S}$ via the leafages $\hat{\alpha}, \hat{\beta}, \hat{\alpha} \circ \hat{\mu}$. Recall that $\mathbb{E}^{\hat{Y}}$ is the set of order-essential edges in $\hat{Y}$. Denote $\mathbb{E}^{Y}:=\Gamma \backslash \mathbb{E}^{\hat{Y}} \subseteq E^{Y}$, and similarly for $\mathbb{E}^{Z}$ and $\mathbb{E}^{S}$. Lemma 2(c) impies that leafages map order-essential edges to order-essential edges, so $\hat{\mu}\left(\mathbb{E}^{\hat{S}}\right) \subseteq \mathbb{E}^{\hat{Y}}$ and $\hat{\nu}\left(\mathbb{E}^{\hat{S}}\right) \subseteq \mathbb{E}^{\hat{Z}}$. Taking the $\Gamma$-quotients yields the diagram

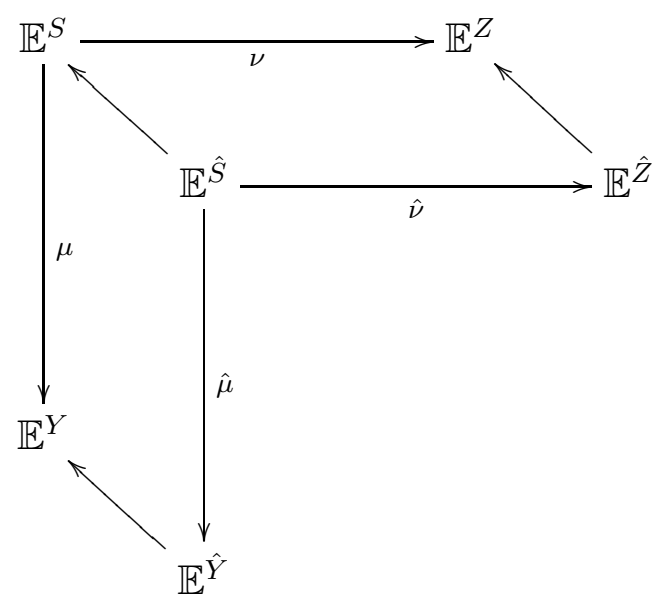

which is part of the system (1).

The proof in a nutshell. $S$ is the fiber product of $Y$ and $Z$ over $X$. In particular, $\mu$ and $\nu$ in (1) induce an inclusion $\varphi: E^{S} \hookrightarrow E^{Y} \times E^{Z}$. Then the restricted versions of $\mu$ and $\nu$ above induce an inclusion $\varphi: \mathbb{E}^{S} \hookrightarrow \mathbb{E}^{Y} \times \mathbb{E}^{Z}$. This implies the inequality $\# \mathbb{E}^{S} \leq \# \mathbb{E}^{Y} \cdot \# \mathbb{E}^{Z}$. By Theorem 5 , $\bar{r}(S) \leq \bar{r}(Y) \cdot \bar{r}(Z)$. This is equivalent to SHNC.

The detailed proof. Consider the diagonal inclusion $\hat{\varphi}: E^{\hat{S}} \rightarrow E^{\hat{Y}} \times E^{\hat{Z}}, \hat{\varphi}(\tau):=(\hat{\mu}(\tau), \hat{\nu}(\tau))$. Explicitly, $\hat{\varphi}(y, z, \sigma):=((y, \sigma),(z, \sigma))$ for $(y, z, \sigma) \in \hat{S}$. Since both $\hat{\mu}$ and $\hat{\nu}$ commute with the $\Gamma$-actions, then $\hat{\varphi}$ induces a map $\varphi: E^{S} \rightarrow E^{Y} \times E^{Z}$ on the $\Gamma$-quotients.

We claim that $\varphi$ is injective. Take any $(y, z, \sigma),(\tilde{y}, \tilde{z}, \tilde{\sigma}) \in \hat{S}$ and suppose that $(\tilde{y}, \tilde{\sigma})=$ $(g y, g \sigma)$ and $(\tilde{z}, \tilde{\sigma})=(h z, h \sigma)$ for some $g, h \in \Gamma$, then $g \sigma=h \sigma$. Since the $\Gamma$-action on $\hat{X}$ is free, $g=h$. This implies $(\tilde{y}, \tilde{z}, \tilde{\sigma})=(g y, g z, g \sigma)$, i.e. $(y, z, \sigma)$ and $(\tilde{y}, \tilde{z}, \tilde{\sigma})$ lie in the same $\Gamma$-orbit. So $\varphi$ is injective.

Now consider only order-essential edges. By Lemma 2(c), the restriction of $\hat{\varphi}$ to $\mathbb{E}^{\hat{S}}$ takes values in $\mathbb{E}^{\hat{Y}} \times \mathbb{E}^{\hat{Z}}$, i.e. gives a map $\hat{\varphi}: \mathbb{E}^{\hat{S}} \rightarrow \mathbb{E}^{\hat{Y}} \times \mathbb{E}^{\hat{Z}}$. By the above argument the induced map on the quotients, $\varphi: \mathbb{E}^{S} \rightarrow \mathbb{E}^{Y} \times \mathbb{E}^{Z}$, is injective. Then $\# \mathbb{E}^{S} \leq \# \mathbb{E}^{Y} \cdot \# \mathbb{E}^{Z}$. By Theorem 5 and Lemma 1, $\bar{r}(S) \leq \bar{r}(Y) \cdot \bar{r}(Z)=\bar{r}(A \backslash \mathcal{A}) \cdot \bar{r}(B \backslash \mathcal{B})=\bar{r}(A) \cdot \bar{r}(B)$. 
It remains to show the equality $\bar{r}(S)=\sum_{u \in s(A \backslash \Gamma / B)} \bar{r}\left(A^{u} \cap B\right)$. Put the left diagonal $\Gamma$-action on the set $(\Gamma / A) \times(\Gamma / B)$ and consider the map

$$
\theta: s(A \backslash \Gamma / B) \rightarrow(\Gamma / A) \times(\Gamma / B), \quad \theta(u):=\left(u^{-1} A, 1 B\right) .
$$

It is clear that $\theta$ is injective. We claim that its image intersects each $\Gamma$-orbit in $(\Gamma / A) \times(\Gamma / B)$ exactly once. Take any element of $(\Gamma / A) \times(\Gamma / B)$, it has the form $(\bar{y} A, \bar{z} B)$ for some $\bar{y}, \bar{z} \in \Gamma$. There exists $u \in s(A \backslash \Gamma / B)$ such that $\bar{y}^{-1} \bar{z} \in A u B$. Hence there exist $a \in A$ and $b \in B$ such that $\bar{z} b=\bar{y} a u$. Then $\bar{z} b \cdot\left(u^{-1} A, 1 B\right)=\left(\bar{y} a u u^{-1} A, \bar{z} b B\right)=(\bar{y} A, \bar{z} B)$, so the $\Gamma$-orbit of $(\bar{y} A, \bar{z} B)$ contains $\left(u^{-1} A, 1 B\right)$. Now suppose $u, \tilde{u} \in s(A \backslash \Gamma / B), g \in \Gamma$, and $g \cdot\left(u^{-1} A, 1 B\right)=\left(\tilde{u}^{-1} A, 1 B\right)$, then $g \in B$ and for some $a \in A, u=a^{-1} \tilde{u} g \in A \tilde{u} B$, hence $u=\tilde{u}$. This shows uniqueness.

The above claim shows that the set $\left\{\hat{S}_{u^{-1} A, 1 B} \mid u \in s(A \backslash \Gamma / B)\right\}$ has exactly one leaf in each $\Gamma$-orbit in Leaves $(\hat{S})$. The stabilizer of the leaf $\hat{S}_{u^{-1} A, 1 B}$ is $A^{u} \cap B$, hence

$$
S=\Gamma \backslash \hat{S}=\Gamma \backslash\left(\bigsqcup_{(y, z) \in(\Gamma / A) \times(\Gamma / B)} \hat{S}_{y, z}\right)=\bigsqcup_{u \in s(A \backslash \Gamma / B)}\left(A^{u} \cap B\right) \backslash \hat{S}_{u^{-1} A, 1 B} .
$$

By Lemma 1, $\bar{r}(S)=\sum_{u \in s(A \backslash \Gamma / B)} \bar{r}\left(\left(A^{u} \cap B\right) \backslash \hat{S}_{u^{-1} A, 1 B}\right)=\sum_{u \in s(A \backslash \Gamma / B)} \bar{r}\left(A^{u} \cap B\right)$.

The same argument proves the following more general result which does not require $\hat{X}$ to be a forest or locally-finite, or the $\Gamma$-action on $\hat{X}$ to be free.

Theorem 6. Suppose $\Gamma$ is any group, $\hat{X}$ is a free-edge ordered $\Gamma$-graph, $\mathcal{A}$ and $\mathcal{B}$ are subgraphs of $\hat{X}, A$ and $B$ are subgroups of $\Gamma$ that stabilize $\mathcal{A}$ and $\mathcal{B}$, respectively, and the actions of $A$ on $\mathcal{A}$ and of $B$ on $\mathcal{B}$ are cocompact. Then

$$
\sum_{u \in s(A \backslash \Gamma / B)} \#\left(\left(A^{u} \cap B\right) \backslash \mathbb{E}^{\left(u^{-1} \mathcal{A}\right) \cap \mathcal{B}}\right) \leq \#\left(A \backslash \mathbb{E}^{\mathcal{A}}\right) \cdot \#\left(B \backslash \mathbb{E}^{\mathcal{B}}\right) .
$$

(Here $\mathcal{A}, \mathcal{B}$ and $\left(u^{-1} \mathcal{A}\right) \cap \mathcal{B}$ are viewed as an ordered $A$-graph, an ordered $B$-graph and an ordered $A^{u} \cap B$-graph, respectively, with the partial orders restricted from $E^{\hat{X}}$.)

If, in addition, $\mathcal{A}$ and $\mathcal{B}$ are forests and the actions of $A$ on $\mathcal{A}$ and of $B$ on $\mathcal{B}$ are free, then

$$
\begin{aligned}
& \sum_{u \in s(A \backslash \Gamma / B)} \bar{r}\left(\left(A^{u} \cap B\right) \backslash\left(\left(u^{-1} \mathcal{A}\right) \cap \mathcal{B}\right)\right) \leq \bar{r}(A \backslash \mathcal{A}) \cdot \bar{r}(B \backslash \mathcal{B}) \quad \text { and } \\
& \sum_{u \in s(A \backslash \Gamma / B)} \bar{r}\left(A^{u} \cap B\right) \leq \bar{r}(A) \cdot \bar{r}(B) .
\end{aligned}
$$

Further, it is possible to remove the assumption of cocompactness if one allows infinite values. In this case the above inequalities still hold with the convention $0 \cdot \infty=0$. 
1.7. Ladders. The ladder of length $m$, denoted $L_{m}$, is the graph consisting of two circles, each subdivided into $m$ edges, and connected consecutively by $m$ edges, as on the picture.

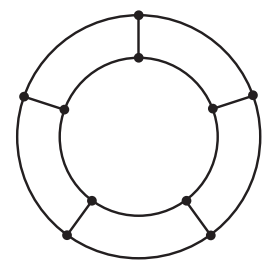

It is easily checked that $\bar{r}\left(L_{m}\right)=m$. For each $m$ there is a covering map $L_{m} \rightarrow L_{1}$ wrapping the two circles in $L_{m} m$ times around the two circles in $L_{1}$. If $m$ and $n$ are relatively prime, $L_{m n}$ is the fiber product of $L_{m}$ and $L_{n}$ over $L_{1}$, as on the picture.

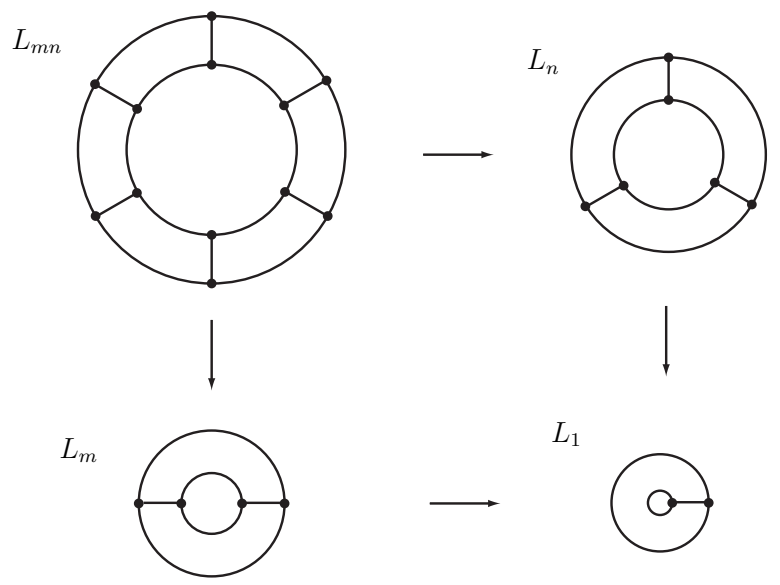

This is a special case of Stallings diagrams [7], and it can be extended to a system as in [1, section 3.1]. We have $\bar{r}\left(L_{m n}\right)=\bar{r}\left(L_{m}\right) \cdot \bar{r}\left(L_{n}\right)$, i.e. the upper bound in SHNC is sharp.

\section{REFERENCES}

[1] I. Mineyev, The topology and analysis of the Hanna Neumann Conjecture, The Journal of Topology and Analysis (JTA), 3 (2011), pp. 307-376.

[2] — Submultiplicativity and the Hanna Neumann Conjecture, Ann. of Math., 175 (2012), pp. 393-414.

[3] H. Neumann, On the intersection of finitely generated free groups, Publ. Math. Debrecen, 4 (1956), pp. 186189.

[4] — On the intersection of finitely generated free groups. Addendum, Publ. Math. Debrecen, 5 (1957), p. 128.

[5] W. D. Neumann, On intersections of finitely generated subgroups of free groups, in Groups - Canberra 1989, vol. 1456 of Lecture Notes in Math., Springer, Berlin, 1990, pp. 161-170.

[6] H. Shimbireva, On the theory of partially ordered groups, Rec. Math. [Mat. Sbornik] N.S., 20(62) (1947), pp. 145-178. Available at www.mathnet.ru.

[7] J. R. Stallings, Topology of finite graphs, Invent. Math., 71 (1983), pp. 551-565.

[8] A. A. Vinogradov, On the free product of ordered groups, Mat. Sbornik N.S., 25(67) (1949), pp. 163-168. Available at www.mathnet.ru. 